\title{
Acque e colture irrigue in Terra di Lavoro tra XVII e XVIII secolo. Una riflessione sul
}

Citation: P. De Felice, L. Spagnoli (2021). Acque e colture irrigue in Terra di Lavoro tra XVII e XVIII secolo. Una riflessione sul paesaggio dell'acqua tra economia e tutela. Bollettino della Società Geografica Italiana serie 14, 4(1): 3-17. doi: 10.36253/bsgi-1241

Copyright: @ 2021 P. De Felice, L. Spagnoli. This is an open access, peerreviewed article published by Firenze University Press (http://www.fupress. com/bsgi) and distributed under the terms of the Creative Commons Attribution License, which permits unrestricted use, distribution, and reproduction in any medium, provided the original author and source are credited.

Data Availability Statement: All relevant data are within the paper and its Supporting Information files.

Competing Interests: The Author(s) declare(s) no conflict of interest.

For Italian evaluation purposes: Luisa Spagnoli takes responsability for sections 1, 5, 6 and Pierluigi De Felice for sections 2, 3, 4 .

\section{Water and crops irrigated in Terra di Lavoro between the seventeenth and eighteenth centuries. A reflection on the landscape of the water between economy and protection}

\author{
Pierluigi De Felice ${ }^{1}$, Luisa Spagnoli ${ }^{2}$ \\ 1 Dipartimento di Studi Umanistici, Università degli Studi di Salerno, Italia \\ 2 Istituto di Storia dell'Europa Mediterranea-Consiglio Nazionale delle Ricerche, Roma, \\ Italia \\ E-mail: pdefelice@unisa.it, luisa.spagnoli@uniroma2.it
}

\begin{abstract}
In the archive of the Abbey of Montecassino there is a judicial affair between the monks of the Abbey and the Duke of Mignano stored. The quaestio of the dispute is for the sowing of rice by the Duke who, despite several orders of prohibitions (1661, 1665), persists in cultivating it, causing, according to the Benedictine monks, "great damage to the universities of St. Vittore, St. Pietro Infine, Mignano" because "it affects the wholesomeness of these lands". An unpublished large-scaled cartography is attached to this judicial dispute, whose graphic signs clarify and define the places of the diatribe also providing further information: we are in the Terra di Lavoro bathed by the river Peccia which is used and partly diverted to irrigate the Duke's rice. The case study offers a lot of food for thought starting from the problematic relationship between the values of the environment and water resources, defended by the Benedictine monks, and the economic and productive needs of a local aristocracy with an entrepreneurial vocation. This contrast connects, recalling and confirming it, to the wider one that developed during the eighteenth and nineteenth centuries linked to the spread of rice where doctors, agronomists, politicians have widely debated the problems raised by the spread of rice fields in the Peninsula.
\end{abstract}

Keywords: cartography, water, reclamation, irrigated crops, environmental protection. 
rapporto tra i valori dell'ambiente e delle risorse idriche, difesi dai monaci benedettini, e le necessità economico-produttive di unaristocrazia locale con una vocazione imprenditoriale. Questa contrapposizione si connette, richiamandola e confermandola, a quella di più ampio respiro che si è sviluppata nel corso del XVIII e XIX secolo, legata alla diffusione della risicoltura dove medici, agronomi, politici hanno ampiamente dibattuto sui problemi sollevati dall'estendersi delle risaie nella Penisola.

Parole chiave: cartografia, acque, bonifiche, colture irrigue, salvaguardia dell'ambiente.

\section{Strumenti per il governo delle acque e la salvaguardia dell'ambiente: uno sguardo d'insieme}

Il patrimonio di risorse territoriali è frutto di un lento processo di accumulazione e sedimentazione, alla cui determinazione ha senz'altro preso parte l'uomo che con la sua azione territorializzante ha modificato la natura. La diversità di azioni messe in campo e la diversità di modelli e approcci culturali hanno generato specifiche identità e valori territoriali che hanno contraddistinto differentemente i singoli luoghi. Il rapporto che gli uomini hanno costruito nel tempo con l'ambiente e le risorse naturali è, dunque, un lungo processo di adattamento e di stratificazioni storiche che ha marcato profondamente i saperi locali, gli atteggiamenti culturali, i comportamenti delle società, dando vita a regole e istituzioni specifiche. Si tratta di un legame di lunga durata - un rapporto co-evolutivo, per dirla con Magnaghi, tra natura e cultura - che ha impresso connotazioni profonde ai territori e alle comunità (Magnaghi 2010). Come rilevava Carlo Cattaneo, è nel legame sinergico tra uomini e ambiente che nascono quei modelli organizzativi non scritti, quelle pratiche quotidiane di lavoro e di vita che nessuna autorità può determinare e imporre. L'acqua con i suoi diversi utilizzi ha da sempre rappresentato una delle risorse più importanti per l'uomo: le società, secondo logiche e modi differenti di agire, sono riuscite nel corso dei secoli a modellare le forme naturali divenute parti costitutive di straordinari paesaggi rurali.

Le produzioni irrigue hanno innescato un rapporto più stretto, più attento e di sorveglianza nei confronti dell'ambiente, che si è esplicitato attraverso modalità di organizzazione e gestione delle acque riconducibili molto di frequente a importanti operazioni di bonifica che hanno caratterizzato l'intera penisola italiana.

E soprattutto in Italia settentrionale e in alcune realtà di quella centrale che si è manifestata un'attenzione alle acque riconducibile specialmente all'azione dei governi comunali e signorili, nonché all'iniziativa di alcune comunità monastiche (Gambi 1989). Una eredità, questa, che si proietta nei dieci secoli successivi influenzando positivamente gli esiti della bonifica ${ }^{1}$.

Se in questa parte della Penisola le opere di regimentazione delle acque hanno assunto forme imponenti sin dall'età moderna, nel Mezzogiorno i primi significativi risultati si hanno solo a partire dal XVIII secolo (Bevilacqua 1986). Diversità soprattutto di situazioni a livello politico, economico e sociale che hanno portato a una netta differenza nella riorganizzazione e gestione idraulica di interi territori.

Da una parte, la capacità di "manipolare" i corsi d'acqua acquisita nel tempo, che ha influito e condizionato anche la vita produttiva, le relazioni sociali e le vicende politiche a tal punto da sollecitare il costituirsi di una vera e propria "economia d'acqua"2 (Cazzola 1987, 37-66); dall'altra parte, soluzioni più sporadiche e certamente non così strutturate e produttive.

È pur vero, comunque, che nell'ambito del dibattito settecentesco il pensiero illuminista che si diffuse nel Regno delle Due Sicilie portò con sé l'idea della centralità del territorio e dell'importanza delle sue risorse. Esisteva, quindi, da parte della spinta riformistica antifeudale un atteggiamento quasi "ecologico", ante litteram, che considerava fondamentali nella società le problematiche di carattere ambientale, l'utilizzo delle risorse, le peculiarità del territorio, le attività produttive, alla luce "di una riforma complessiva della società" (Bevilacqua 1989a, 74; Manzi, 1987; Foscari 2009)³. Questo atteggia-

${ }^{1}$ La risposta della pianura settentrionale alle problematiche idrografiche è soprattutto una risposta delle comunità al problema dello sviluppo dell'agricoltura e del miglioramento delle tecniche d'intervento per una più significativa produttività nel campo agricolo.

${ }^{2}$ È così che nell'Italia Padana, in ragione certamente dell'abbondante disponibilità di acque, si diffusero e sedimentarono nel corso del tempo consuetudini giuridiche, istituti, atteggiamenti, "visioni" per la difesa delle terre e per l'utilizzo collettivo delle acque (Bevilacqua 2000).

${ }^{3}$ Molti in questo senso gli scrittori "illuminati" che auspicarono per il Mezzogiorno un risanamento dei territori e delle condizioni economico-produttive, tra cui Carlo Afan de Rivera alla direzione del Corpo di Ponti e Strade, Acque, Foreste e Caccia del Regno delle Due Sicilie, che andò caldeggiando una programmatica opera di bonifica per tutti i territori del Regno (Di Bisio 1991; Foscari 1992). In un passo della sua opera, Afan de Rivera si sofferma sui vantaggi che le acque "regolamentate" avrebbero comportato per la provincia di Terra di Lavoro, da S. Germano alla foce del Garigliano: un grande beneficio in termini di produttività dell'agricoltura e dell'industria. "Bonificate le pianure contigue al fiume, la cui fertilità si può aumentare per mezzo delle irrigazioni, [...], l'agricoltura, la pastorizia, l'industria ed il commercio potrebbero fare i più grandi progressi e favoreggiarsi a vicenda. Il grosso volume delle acque del Garigliano animando numerosi motori idraulici, irrigando una vasta superficie di fertilissime terre, ed apprestando una facile comunicazione per acqua, acquisterebbe un prodigioso valore" (Afan de Rivera 1833,6$)$. Al di là di problematiche specifiche relative al miglioramento di determinati quadri ambientali, le idee riformiste furono particolarmente sentite nel Regno delle Due Sicilie. Il movimento ispirato 
mento, dunque, che implica una sollecitazione da parte di forze sociali più dinamiche indirizzate alla cura e al risanamento dell'ambiente, alla comprensione del nesso fra degrado ambientale e degrado economico, morale e culturale, è esistito ed è stato condiviso da una parte degli studiosi riformisti ${ }^{4}$.

Anzi, tali esigenze, come sottolinea Bevilacqua (1989a), non dovevano essere in contraddizione con lo sforzo "di risanamento del Regno e di sviluppo economico generale" che, dopo la Restaurazione, si è esplicitato attraverso tentativi di bonifica qua e là disseminati, in maniera non uniforme, sul territorio del Mezzogiorno (Ciasca 1928; D’Elia 1994; Foscari 2009; Conti et al. $2010)^{5}$. Qui l'intervento dello Stato dovette essere più incisivo e intenso dovendo farsi carico di un notevole sforzo economico ${ }^{6}$, a dispetto di quanto avveniva in Italia settentrionale in cui furono principalmente i privati a investire nelle coltivazioni irrigue e, quindi, nelle opere di governo delle acque, creando e sperimentando

\footnotetext{
all'Illuminismo cominciò a diffondersi nel Mezzogiorno d'Italia intorno alla prima metà del Settecento, soprattutto alla luce della conquista da parte di Carlo di Borbone del Meridione, decretando la fine del governo austriaco. Le riforme avviate da Carlo furono proseguite in parte sotto il figlio Ferdinando IV, in virtù dell'azione politica espressa dal ministro reggente Bernardo Tanucci. Non solo, a formare il pensiero illuminato del nuovo stato indipendente contribuì certamente il giurisdizionalismo di Pietro Contegna, Alessandro Ricciardi, Costantino Grimaldi, Pietro Giannone, così come le idee di Giuseppe Maria Galanti, Ferdinando Galiani e del già citato Carlo Afan de Rivera, interpreti di una visione incentrata sulla necessità dell'attuazione di una più analitica conoscenza territoriale, i cui "sogni geoterritoriali-cartografici" si concretizzarono nella feconda attività cartografica di Rizzi Zannoni (Manzi 1987, 349). Idee, sogni, ambizioni di una politica economica e sociale autonoma, indirizzata a riformare la compagine statale, si diffusero nel Napoletano, sfociando successivamente nel giacobinismo meridionale e nella rivoluzione del 1799.

${ }^{4}$ Tale atteggiamento in Afan de Rivera si esplicita soprattutto come attenzione nei confronti del legame diretto fra mancanza di cura della montagna e dissesto idrogeologico.

${ }^{5}$ Durante tutta la prima metà dell'Ottocento si registrò uno sforzo senza precedenti da parte dei tecnici e dell'amministrazione napoletana per corredare lo stato di una legislazione organica in materia di bonifica che portò alla creazione, l'11 maggio del 1855 , dell'Amministrazione generale delle bonificazioni, la cui attività fu interrotta dall'Unità d'Italia. Nel 1864 l'Amministrazione generale, infatti, fu sciolta mettendo fine a un organismo che, sebbene perfettibile, era comunque uno strumento moderno al servizio degli interessi pubblici. L’uniformità normativa del neo Stato unitario "liquidava il frutto di una sapienza tecnica e insieme di una giurisprudenza, che si erano sforzate di aderire ai caratteri originali dell'ambiente meridionale, alla sua particolare storia, e che tendevano a svolgere, per la prima volta, un ruolo di attivo governo sulle forze produttive operanti nel territorio" (Bevilacqua 1986, 357).

${ }^{6}$ Ricorrere all'acqua per fini irrigui, utilizzandola su vasta scala e sistematizzandola in forme regolate, non sarebbe stato fattibile senza un riordino complessivo dell'intera rete idrografica meridionale. Un'impresa, questa, che non poteva che rinviare a un compito di ampie proporzioni di esclusivo appannaggio statale (Bevilacqua 1986, 339).
}

consorzi irrigui per la loro gestione $\mathrm{e}^{7}$. Il governo del territorio esplicitato attraverso l'opera di bonifica dovette incarnare una volontà politica che non trovò spesso l'appoggio da parte della società civile, disattendendo lo spirito innovativo e lungimirante dello Stato e dei suoi tecnici (Bevilacqua 1986). Esemplari, a tale riguardo, le parole di Afan de Rivera: "Certamente non può esservi oggetto di maggior comune vantaggio come quello di liberare dall'infezione dell'are vaste contrade, ove a migliaia di uomini cadono vittime del bisogno di guadagnarsi la vita con le proprie fatiche che loro non riesce d'impiegare altrove" (Afan de Rivera 1833, 144-145).

Questa la cornice di riferimento per sollecitare una riflessione sul complesso rapporto che nel tempo si è costruito tra comunità e risorse idriche in una porzione della provincia di Terra di Lavoro. Si è trattato di leggere e interpretare una corposa documentazione testuale e un'inedita cartografia a grande scala; testimonianze conservate nell'archivio dell'Abbazia di Montecassino, concernenti una contesa giudiziaria relativa alla coltura irrigua del riso, tra l'Abbazia stessa e le università di $S$. Vittore, S. Pietro Infine (in Terra di Lavoro), da una parte, e, il Duca di Mignano, dall'altra. Montecassino estendeva il suo dominio nella "Terra di San Benedetto", che era ricompresa nell'antica provincia di Terra di Lavoro. Quest'ultima coincideva con le odierne province di Latina e Frosinone (nel Lazio), con le attuali province campane di Caserta, Benevento e parte di Napoli e con quella di Isernia (Venafro). Al 1860 "La provincia di Terra di Lavoro era costituita da 5 distretti (Capua, Gaeta, Nola, Piedimonte e Sora) e contava ben 234 comuni. Nelle province del Regno delle Due Sicilie non era contemplata quella di Benevento che, sino al 1860, era un'exclave dello Stato Pontificio all'interno del Regno" (Conti 2009, 324).

I suoi confini variarono nel corso dei secoli e, tra Otto e Novecento, la provincia fu interessata da un generale riassetto del territorio che ne decretò la scomparsa e definitiva soppressione con il regio decreto del 2 gennaio 1927, n. 1 (Conti, Di Biasio 2012; Franzese 2020). È in una parte di questo territorio, caratterizzata da zone di impaludamento utilizzate prevalentemente per la coltivazione del riso, che prende corpo la già menzionata vicenda giudiziaria. Come si leggerà nelle pagine successive, il ricorso a tale tipo di coltura provocò non pochi problemi nel Meridione, così come nell'Italia settentrionale, a causa dei miasmi che ne derivavano, a tal punto da indurre

\footnotetext{
${ }^{7}$ A differenziare ulteriormente il quadro va sottolineata la mancanza nel sud della Penisola di una rete produttiva che nelle città trovava la propria ragione d'essere: siamo spesso in presenza nei territori rurali di ampi possedimenti degradati sotto il profilo idraulico che avevano come unici punti di riferimento sul territorio insediamenti di limitate dimensioni e carenti dal punto di vista tecnico e culturale (Bevilacqua 2000).
} 
le autorità locali a prendere provvedimenti per regolare l'uso delle risaie e il loro posizionamento che doveva rispettare una certa distanza dai centri abitati, che era comunque variabile a seconda dei contesti (infra).

Travalicando i confini della lite, la rappresentazione cartografica - con la sua innegabile dimensione euristica fondamentale per la ricostruzione dei quadri territoriali del passato - e l'annessa documentazione invitano a ripensare il "significato" di quei paesaggi irrigui che sono venuti delineandosi nel corso del tempo, connotando i territori dell'Italia meridionale solo tra Sette e Ottocento. La valorizzazione di tali testimonianze consente, dunque, di far emergere le stratificazioni profonde materiali e valoriali sedimentatesi nel territorio, con la finalità ultima di individuare e prefigurare strategie d'intervento su di esso.

\section{La contesa giudiziaria tra il monastero di Montecassino e il Duca di Mignano: una riflessione geostorica sulla risicoltura nei secoli XVII-XVIII}

Nel XVII secolo il monastero di Montecassino e i Comuni di San Pietro Infine, Cervaro e San Vittore adiscono legalmente contro il Duca di Mignano, Don Francesco di Capua, per la "semina de' risi" nella località le Starze, alle falde del Monte Lungo in Terra di Lavoro, dando vita ad una lunga e controversa contesa giudiziaria che si protrasse per ben 52 anni (1661-1713).

I documenti inediti ${ }^{8}$ ritrovati nell'Archivio dell'Abbazia di Montecassino (d'ora in poi AAM), insieme alla cartografia storica, commissionata per individuare il luogo della coltivazione del riso e la sua posizione rispetto ai centri vicini, permettono di ricostruire, da una parte, il paesaggio agrario storico della provincia di Terra di Lavoro confinante con i possedimenti della Terra Sancti Benedicti ${ }^{9}$ a cavallo fra il XVII e XVIII secolo, dall'altra

\footnotetext{
${ }^{8}$ Nell'Archivio dell'Abbazia di Montecassino (AAM), per la ricostruzione della contesa giudiziaria sulla risaia di Mignano abbiamo consultato la capsula CXXXIV Fasc. II Scripturae extractae a processibus causae orizarum inter Sacru(m) Monasteriu( $m$ ) Casinense et Ill.m Ducem Mignani e la capsula CXXXIV Fasc. III Scritture Spettanti alla lite tra il Sacro Monastero Cassinese e 'l Signor Duca di Mignano per la semina dérisi dal 1661 al 1713 in cui si venne a transazione. Il riferimento alla capsula rimanda alla catalogazione dei secoli XVI e XVII quando i documenti dell'Archivio di Montecassino vennero trasferiti dai sacchi alle capsule o cassetti. Questa sistemazione è durata fino agli inizi del sec. XIX. I faldoni si compongono di diversi documenti (relazioni, strumenti, corrispondenze) che abbiamo citato facendo riferimento allintestazione del documento e al numero del folio se è segnato. Per la storia dell'Archivio di Montecassino e della sua consistenza si confronti Archivio dell'Abbazia di Montecassino (1986).

${ }^{9}$ Per una ricostruzione dellestensione della Terra di San Benedetto e dei suoi possedimenti si veda Fabiani (1968) che ne ricostruisce i confini a partire dalla donazione di Gisolfo II.
}

parte, restituiscono informazioni rispetto alla gestione del territorio rurale sia per quanto concerne l'organizzazione produttiva ed economica sia per la gestione delle risorse idriche che vengono utilizzate per la risicoltura.

Dalla dialettica giudiziale, arricchita dalle relazioni dei medici e di "esperti", chiamati dai giudici e dai contendenti a pronunciarsi sugli impatti territoriali della risicoltura, emerge, anche, una particolare attenzione verso i quadri ambientali e sociali, espressione di una sensibilità ecologica ante litteram.

I termini della contesa sono da rintracciarsi ufficialmente proprio negli effetti negativi derivanti dalla coltivazione del riso, che pregiudica la qualità dell'aria, le colture e la salute della popolazione locale. Si legge nei documenti che "il seminato de' Risi introdotto dal Sig. Duca di Mignano apportava gran danno oltre a frutti, alla salute et alle vite de Cittadini di dette terre per essere il Campo delli Risi in luogo aperto, in modo che non vi era riparo che impedisse il vento acciò non portasse la pestifera esalazione che sorgeva dall'acque che lentissimamente scorrevano per qualche tempo e poi nel tempo della ricolta totalm.te stagnavano e dalla putreffazzione (sic!) di tante immundizie e di tanti animali sino alle menzionate terre i di cui abitanti per così pessima esalazione o vedeansi languire oppressi tutti da somigliante et uniforme morbo o si vedevano miseramente perire" (AAM, Capsula CXXXIV, fascicolo III, Stromento per la semina de' Risi, f. 2, recto).

In seguito alla denuncia presentata dal monastero di Montecassino e dai comuni confinanti con il territorio dove insisteva la risaia, il presidente della Regia Camera della Sommaria ${ }^{10}$, Carlo Calà, nel 1661 intima al Duca di Mignano, con una Provisione, di estirpare la risicoltura.

Questo provvedimento non ebbe alcun effetto tanto che nel 1665 sia il monastero che i comuni interessati, alla luce del fatto che il Duca di Mignano, imperterrito, continuasse a coltivare il riso, chiesero e ottennero un nuovo provvedimento, supportato anche dalle dichiarazioni di medici e testimoni ${ }^{11}$ confermando che il riso: "ritrovandosi seminati subito fussero levati e stirpati dalli Padroni di detti seminati e non facendolo fusse

\footnotetext{
${ }^{10}$ Istituita nel Regno di Napoli nel XII secolo e rimasta in vita fino al 1806, la Regia Camera della Sommaria fu un organo di revisione di tutti i conti dello Stato e delle università nonché tribunale per i contenziosi di tipo amministrativo.

11 Alcune di queste dichiarazioni risultarono poi essere fallaci: "del Sig. Duca dal medesimo si diede il termine della ripulsa per poter far conoscere non meno la falsità dei detti testimonij esaminati per parte del monastero et Università ma anco per fare apparire che quelli erano sospetti ad esso Sig. Duca in guisa che non potevano meritare veruna fede e questa replica fu cagione che i P.P. non avessero parlato di questa causa per lo spazio d'anni vent'uno" (AAM, Capsula CXXXIV, Stromento per la semina de' Risi, fascicolo III, f. 12, recto).
} 


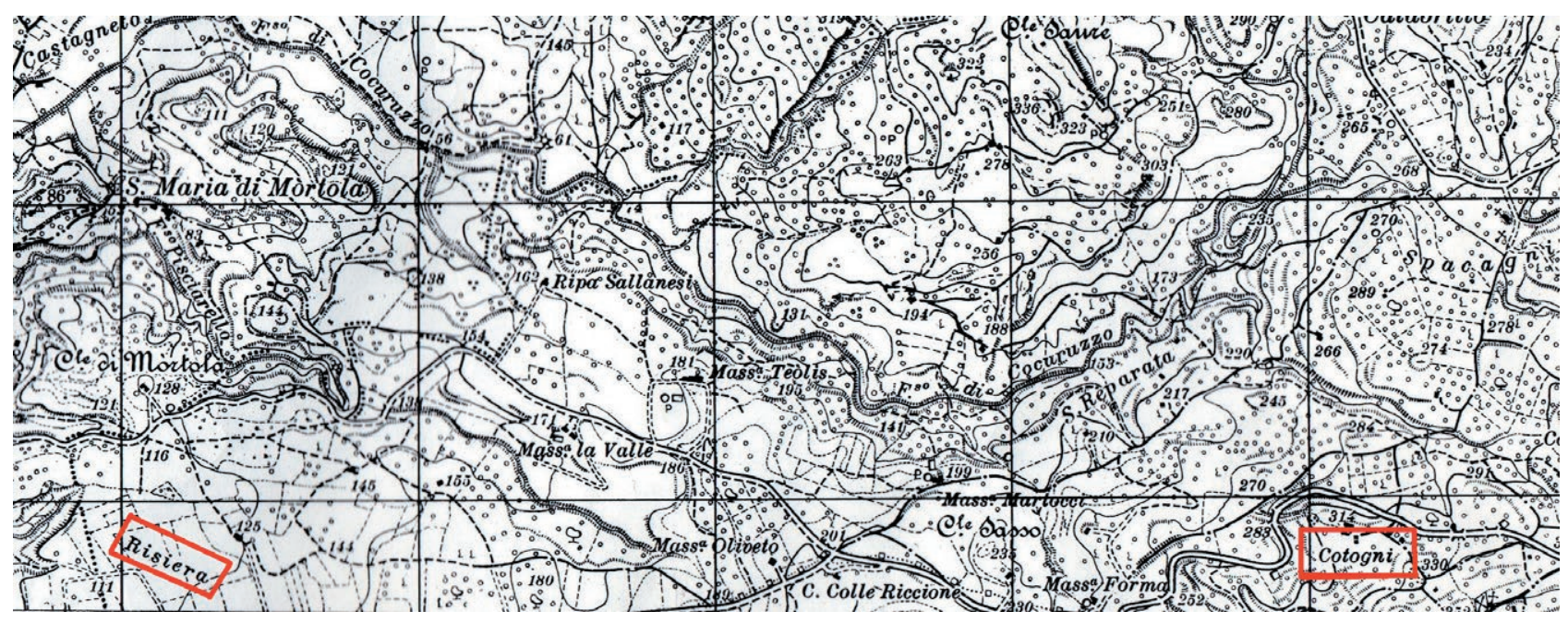

Figura 1. Stralcio della Tavoletta "S. Ambrogio sul Garigliano" (F. 160, II S.E., 1942) dove insistono i toponimi di Risiera e di Cotogni, testimonianza della risicoltura nel comune di Galluccio nei secoli XVII e XVIII. Fonte: Istituto Geografico Militare.

lecito alli Gover.ri e luogoten.ti di dette terre di levarli e stirparli a loro piacere e così si facesse osservare sotto gravissime pene" (AAM, Capsula CXXXIV, fascicolo III, Stromento per la semina de' Risi, f. 2, recto).

Nel 1668 si ripropose la vexata quaestio. L'abate di Montecassino si rivolse direttamente al Vicerè, Don Pietro Antonio d'Aragona, affinché dirimesse la complessa questione dell'impatto ambientale e territoriale della risicoltura. In questa occasione il Governatore di Capua, avvalendosi della consulenza di medici ed 'esperti', tra cui un "misuratore" per la realizzazione di una pianta del luogo, raccolse ulteriori prove e testimonianze. Quest'ultime furono in toto contestate dal Duca di Mignano che continuò, irremovibile, la sua attività ampliando anche la superficie coltivata. Attraverso circostanziate relazioni ${ }^{12}$ il Duca ridimensionò gli effetti negativi e depotenziò le motivazioni addotte dai monaci e le deposizioni dei testimoni, alcune delle quali ritenute false.

Dopo un silenzio durato circa 13 anni, i monaci ricorsero nuovamente nel 1681 contro il Duca. In questa occasione ulteriori ricognizioni, insieme alla stesura di relazioni e all'elaborazione di una cartografia, chiarirono maggiormente la complicata questione della gestione delle risaie di Mignano Montelungo.

La lite si concluse agli inizi del XVIII secolo con un

\footnotetext{
${ }^{12}$ In AAM, Capsula CXXXIV, fascicolo III è conservato un documento dal titolo: "Difesa della cultura del riso che si fa nel territorio di Mignano" commissionata dal Duca di Mignano ad undici medici che in quattro capitoli contestano le motivazioni sanitarie e ambientali addotte dai Monaci e dalle Università riguardo alla risicoltura. Il documento offre una testimonianza delle conoscenze possedute nel XVIII secolo in Italia meridionale riguardo agli effetti ambientali e sociale della risicoltura.
}

accordo tra le parti consistente in un risarcimento in denaro al Duca di Mignano da parte del monastero di Montecassino e dei Comuni coinvolti nella querelle giudiziaria affinché sospendesse la coltivazione del riso nei territori di Mignano. Azione che compromise definitivamente la pratica della risicoltura in quel territorio facendo scomparire ogni segno di questa attività agricola che oggi resiste, seppur in modo flebile, solo nella "coscienza territoriale" locale.

Questa pratica colturale, inoltre, era presente non solo a Mignano Montelungo ma anche in altri comuni vicini, grazie alla importante idrografia continentale locale (fiume Garigliano). Ne ritroviamo conferma nei documenti d'archivio che attestano la coltivazione del riso in altri comuni vicini come, ad esempio, quello di Galluccio il cui Barone aveva impiantato una risaia nei pressi della contrada di Cotogni, toponimo che ancora è presente nella cartografia IGM insieme a quello di "risiera" (Fig. 1). Ulteriori testimonianze delle attività risicole si registrarono nei secoli XVII e XVIII nei comuni di Vairano, Venafro, Mondragone.

L'avversità dei Benedettini verso la risaia di Mignano trova la sua giustificazione, oltre che nelle tanto ostentate azioni di tutela e salvaguardia del territorio e della popolazione locale, anche negli interessi economici privati, legati alla presenza di un vasto possedimento di proprietà dell'Abbazia di Montecassino, attiguo a quello del Duca di Mignano.

Questo terreno, denunciavano i religiosi, non veniva coltivato dai contadini "per lo manifesto pericolo di perdere la salute e per la scarsezza degli abitatori delle terre vicine caggionata dalla semina de' Risi" (AMM, 
Capsula CXXXIV, fascicolo III, Stromento per la semina de' Risi, f. 8 recto). Il ripristino, secondo i monaci, delle precedenti colture quali l'orzo, il grano e il miglio avrebbe garantito non solo un cespite sicuro, ma, anche, una migliore qualità ambientale del luogo.

La documentazione commissionata dal Duca, al fine di rispondere in modo puntuale alle diverse accuse mosse dai monaci e dai Comuni vicini, ci informa, dettagliatamente, sull'organizzazione di questa risaia, sulla sua posizione geografica e sul suo funzionamento, restituendoci la narrazione di un'attività imprenditoriale agricola locale innovativa, rispetto al contesto, ed espressione di una vivacità economica che intimoriva i monaci, attenti a custodire gli interessi di quel ricco patrimonio, costituito dalla Terra Sancti Benedicti, da tutelare e valorizzare.

La contrapposizione tra il duca di Mignano e il monastero di Montecassino diventa un vero e proprio topos che contraddistinguerà i secoli successivi vedendo contrapporsi, da una parte, la risicoltura e, dall'altra, il rischio per l'ambiente e la salute dell'uomo.

\section{La "seminazione delli Risi" a Mignano Montelungo}

Il Duca aveva impiantato la risaia nella campagna di Mignano Montelungo, a nord della provincia di Caserta, tra il Monte Lungo (Fig. 2) e il Monte Moscuso, di natura calcarea, in quel lembo di pianura alluvionale dove scorre il fiume Peccia che, originatosi nel comune di Galluccio, diventa un affluente del Garigliano nei pressi del Comune di San Vittore (Fig. 5).

Proprio questo fiume, nel 1659, viene canalizzato in località Starze dal Duca di Mignano, per portare l'acqua a un campo destinato alla coltivazione del riso.

A tal proposito Giustiniani, pur attento osservatore, si limita ad annotare che Mignano "è situata su di un picciol fiume, che le cagiona niente buon'aria. Gli abitanti ascendono a circa 800 addetti alla semina, ed alla pastorizia" (Giustiniani 1803, 17).

In questo territorio, con una chiara vocazione agricola legata ai seminativi e all'allevamento, il Duca di Mignano introduce l'innovativa, per l'epoca e per il luogo, coltura del riso. I documenti d'archivio ci informano, dettagliatamente, anche sulla tecnica colturale della risicoltura restituendoci l'immagine di un'agricoltura che tenta di svilupparsi e innovarsi rispetto ad una tradizione agricola fortemente ancorata alla cerealicoltura e, in parte, ancora molto arretrata, considerati anche gli strumenti agricoli utilizzati (Di Biasio 1979).

Il terreno destinato alla risicoltura, prima di essere riempito d'acqua, veniva più volte arato affinché si rendesse soffice. Una volta trasformato in limo, si formava-

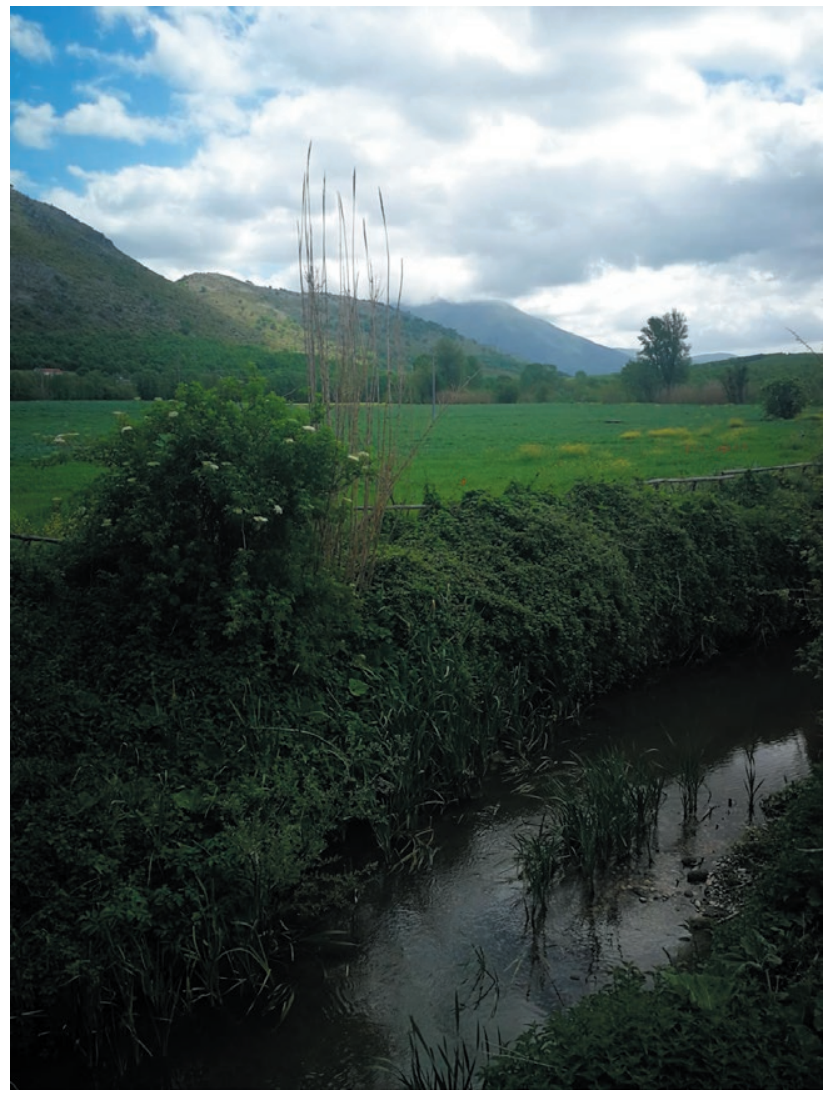

Figura 2. Il Fiume Peccia, in località Moscuso, all'ombra del Monte Lungo dove si trovava la risaia del Duca di Mignano. La memoria storica locale riconosce questo luogo come "Risiera". Fonte: fotografia degli autori.

no dei solchi delimitati da ciglioni e inondati dalla risorsa idrica che veniva fatta entrare attraverso un apposito foro. In seguito, entravano i buoi che attraverso l'ausilio di un erpice - la fonte lo definisce volgarmente manga$\mathrm{no}^{13}$ - aravano il terreno. Dipoi, intervenivano gli uomini che rompevano le zolle di terra (nei documenti sono identificate come glebbe, ciotte, zeppe, cienche). Seguiva poi la semina del riso e, una volta seminato, sia i buoi sia i contadini li ricoprivano con la terra inondandoli di acqua, così da permetterne la germogliazione. Per favorirne la mondatura veniva chiusa momentaneamente la "boccarola" che era il canale da cui proveniva l'acqua per la risaia, così da consentire alle donne scalze di entrare nel campo "per nettare il riso alzando e ponendo sopra i ciglioni tutto ciò che si trova d'immondezza e d'erbe o inutili o dannose al Riso" (AMM, Capsula CXXXIV, fascicolo III, Stromento per la semina de' Risi, f. 6, recto).

${ }^{13}$ Strumento di legno di forma rettangolare costituito da denti che viene tirato dai buoi per spianare e tritare la terra. 
Per garantire la semina e il raccolto del cereale viene confermata l'importanza della fonte idrica che deve essere perennemente garantita: "l'acqua adunque è perpetuamente necessaria a tal coltivazione anzi fa bisogni che di continuo si tramandi nel campo de' Risi [...] che in detti tempi mancasse e mancasse per due o tre giorni la seminazione de' Risi sarebbe affatto perduta" (ibidem).

I monaci e le universitates si opposero a questa procedura, canonica rispetto alla tradizione risicola, perché: "cadono le frondi, le foglie e i fiori della stessa Biada le quali in essa (cioè l'acqua) si corrompono e si putrefanno e dentro dell'acqua anch'essa corrotta e putrefatta si genera ancora infinito stuolo di rane, rospi et anche serpi e quantità tavani o vero serrapichi et altri animaletti armati di pungiglioni i quali poi mandando l'acqua muoiono in gran parte; onde dal mischiamento di quei corpiccioli morti col limo, col fango e coll'altre immondezze che sono in quel campo ne sorgono pessime esalazioni e vapori si che l'aria d'intorno se ne rende di pessimo odore" (AMM, Capsula CXXXIV, fascicolo III, Stromento per la semina de' Risi, f. 7, recto).

Queste motivazioni presentate dalle parti avverse, corroborate da "esperti" e testimoni, ci informano anche sul livello di conoscenza che si aveva tra il XVII e XVIII secolo rispetto alle problematiche legate alle aree mefitiche. Le acque stagnanti, la putrefazione degli animali, le esalazioni "corrompono e infettano l'aria". Il vettore dell'infezione viene individuato erroneamente nell'aria e vengono ad essere colpevolizzati i venti che esalano le pestifere evaporazioni. Viene incriminato anche il periodo della concimazione e della lavorazione dei campi risicoli che corrisponde alla stagione estiva, dove a causa delle alte temperature si registra una "debolezza de' corpi, cagionata dal gran calore”, per cui "l'humana salute soggiace più facilmente a morbi" (ibidem).

Si fa memoria nei documenti giudiziari anche degli effetti territoriali scaturiti dalla risicoltura: la Terra di Mastrati (centro in Terra di Lavoro), un tempo "populatissima divenne spopulata affatto doppo che incominciò vicino ad essa a seminarsi il riso"; così come dei centri di Cotugni e Marotta nel Comune di Galluccio "non è restato altro che ruina" (AMM, Capsula CXXXIV, fascicolo III, Stromento per la semina de' Risi, f. 3, recto).

La distanza da questi luoghi diventa sicuramente un elemento di garanzia per le parti avverse dal rischio di infezioni e patologie. Quante miglia dalla risiera del Duca di Mignano avrebbero garantito la sicurezza? Come si presentava il territorio? Quali culture insistevano su quelle terre? Per rispondere a queste domande si rendeva necessario, in primis, calcolare lo spazio assoluto e, poi, attraverso un'attenta ricognizione sul luogo, rappresentare il paesaggio nei suoi elementi naturali e antropici. Operazione, quest'ultima, che venne affidata nel 1668 al "tavolario" Domenico Sabbatini che "si conferisse sopra la faccia del luogo per poter formare la relazione e pianta delle risiere e delle terre convicine" (AMM, Capsula CXXXIV, fascicolo III, Stromento per la semina de' Risi, f. 10, recto) e nel 1685 a Luise Nauclerio che, insieme al reggente della Regia Camera della Sommaria, agli avvocati e ai medici si recò in quei luoghi dove "si fecero vari atti e varie sperienze e ricognizioni" (AMM, Capsula CXXXIV, fascicolo III, Stromento per la semina de' Risi, f. 12 , recto).

\section{4. "Per avere la notizia dei luoghi con la loro distanza si fece formare la pianta". Il potere della rappresentazione cartografica nella controversia giudiziaria}

Nel fondo Piante e Carte dell'Archivio dell'Abbazia sono conservate due carte, catalogate come "Mignano Piantagione Riso M 8", segnatura recente che sostituisce quella antica che recita "n. V caps. 134 Pianta delli Risi di Mignano fatta nell'anno 1668" (Fig. 3) e "Mignano M 9" (Fig. 4), che subentra alla notazione seicentesca "Pianta delli terreni di Mignano dove si seminano li Risi n. V caps. 134".

Un tempo le carte erano annesse all'affaire giudiziario. Una successiva sistemazione dell'archivio ha separato i documenti dalla cartografia rendendo spesso muta la produzione cartografica. Nel nostro caso a partire dalle carte siamo risaliti alla documentazione archivistica che ci ha permesso di conoscere il fine della produzione di questa cartografia, i committenti, i cartografi che l'hanno prodotta e il periodo di esecuzione.

Lo studio delle diverse fonti d'archivio ha permesso di attribuire la produzione cartografica, elaborata come documento d'appoggio alle incresciose questioni giudiziarie legate alla risicoltura, ai "tavolari" Domenico Sabbatini e Luise Nauclerio.

Queste rappresentazioni contribuiscono a chiarire la questione della risicoltura, promossa dal duca di Mignano, e gli impatti ambientali, territoriali e sociali, scaturiti da questa pratica colturale.

Il Sabattini si recò a Mignano Montelungo e "fe' la Relazione e la pianta ordinatali", rappresentando alcuni dei centri abitati coinvolti (S. Vittore e S. Pietro Infine); la rete stradale (strada Reale); l'idrografia fluviale, disegnando sia il fiume principale (Peccia) che i suoi affluenti (Rio di San Vittore, Rio Vernile, Ripa Rosse); l'orografia (Monte Sant'Angelo, Sambucolo, oggi denominato Sambucaro, Monte Rotundo, Monte Moscoso, oggi Moscuso, Peschito, Cesamo, Monticello, Monte Longo, oggi Monte Lungo); la vegetazione e, infine, la risaia che 


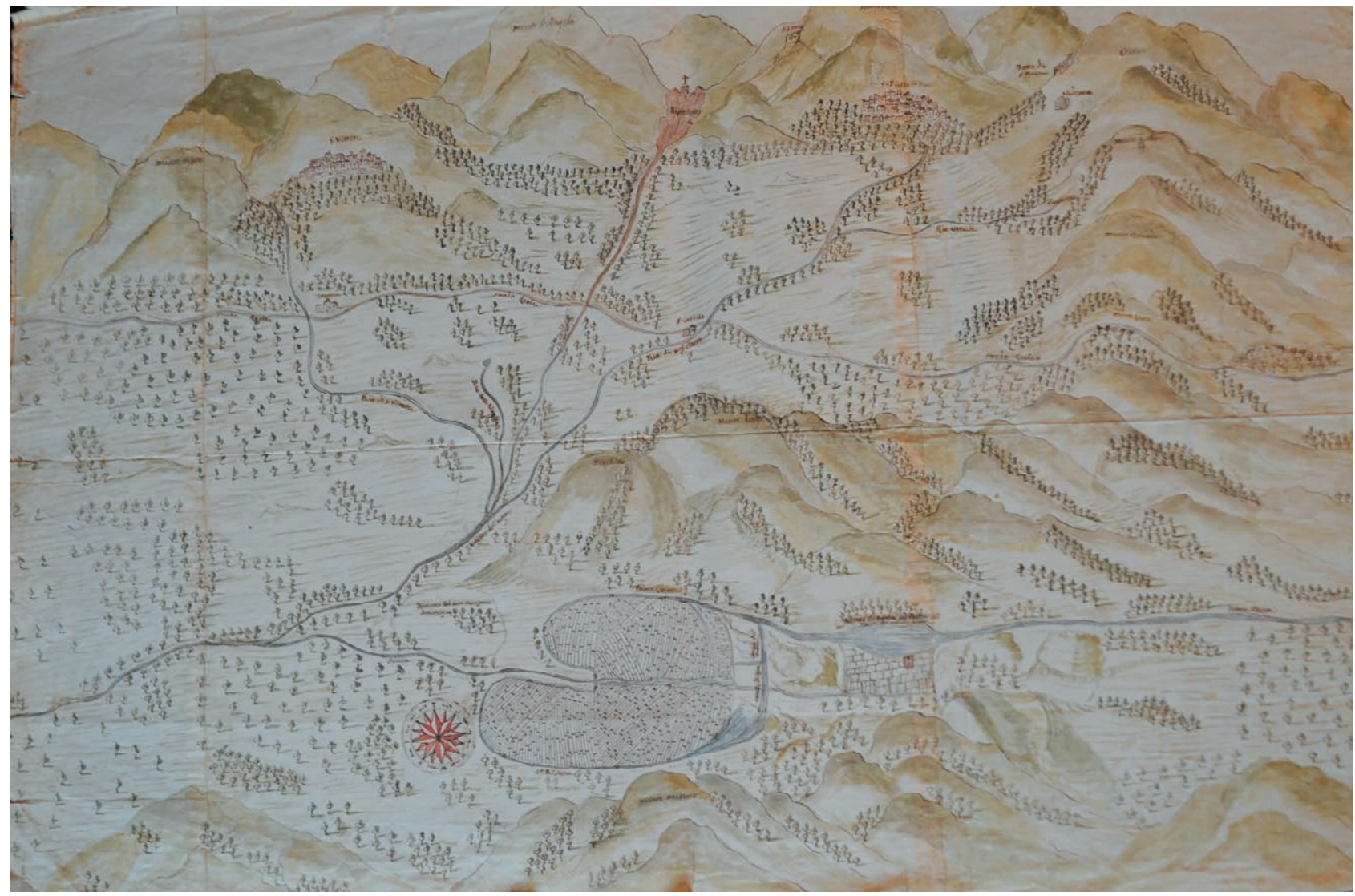

Figura 3. Domenico Sabbatini, Pianta delli Risi di Mignano fatta nell'anno 1668. Carta acquerellata (54 x $81 \mathrm{~cm})$. Fonte: AMM, Carte e piante, M. 8 .

domina lo spazio diventando un iconema del paesaggio rurale. Viene anche disegnato il terreno di proprietà del Monastero di Montecassino, contiguo alla risaia del Duca, confermando l'interesse economico dei monaci nel proteggere quegli ettari, garanti di cespiti.

La rappresentazione del terreno destinato alla risicoltura ("Riparo di fabbrica seu parata dell'acqua che va alle risiere", "canale di fabrica" (sic!), "canale di legno", "prima risiera", "seconda risiera") ci restituisce, anche, la tradizione colturale e l'organizzazione strutturale della piantagione, confermando lo stretto rapporto risorsa idrica/coltivazione.

Nel 1685 fu la volta del "Tavolario" Luise Nauclerio che insieme alla carta (Fig. 4) consegnò anche la relazione dando conto delle attività e dei sopralluoghi resisi necessari per la realizzazione della cartografia, strumento strategico per dirimere la questione giudiziaria (AMM, Capsula CXXXIV, Relat.ne del Tavolario per li risi di Mignano). Si reca a Mignano e precisamente nei pressi del fiume della Peccia, riconoscendo in esso la fonte idrica che alimentava la risaia; segna la montagna di Monte Lungo che si erge non lontano, distinguendo l'altezza "dei ripari de' Risi” e misurando la quantità "dell'acqua d'un quadro de' Risi".

Il suo compito era di elaborare, in origine, due piante "una del campo de' Risi a parte con distintione di tutte le cose" e un'altra "di d.o Campo de' Risi con le montagne che lo circondano in particolare [...] delle montagne di Peschito e Monte Longo che si impongono fra il campo de' Risi e le dette terre di S. Vittore, S. Pietro Infine e Cervaro con misurare l'altezza de montagne e distinguere l'alberi e loro altezza, qualità dell'erbe che nascono in d.e montagne; delinearsi anche il sito della Terra di Mignano, Cervaro e M.te Cas.no e misurare per linea retta la distanza di tutti detti luochi" (AMM, Capsula CXXXIV, Relat.ne del Tavolario per li risi di Mignano). Questa progettualità venne, poi, ridimensionata $\mathrm{e}$ l'attento Nauclerio si limitò a rappresentare lo status quo del territorio risicolo solo attraverso una elaborazione cartografica (Fig. 4).

La carta del Nauclerio risponde pienamente alle istanze giudiziali e la sua lettura ci permette, da una 


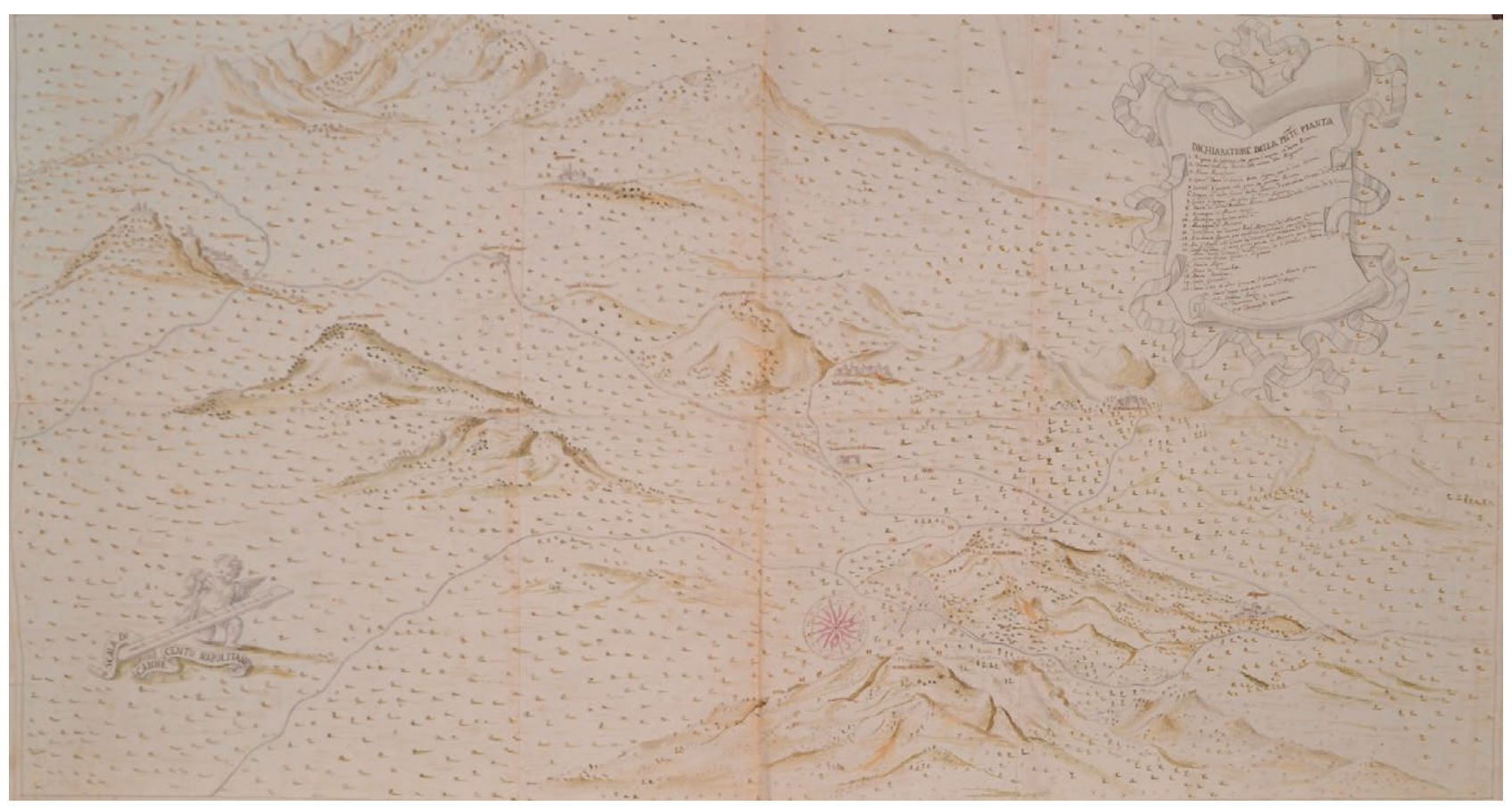

Figura 4. Luise Nauclerio, Pianta delli terreni di Mignano dove si seminano li Risi, 1685. Carta acquarellata (76 x $142 \mathrm{~cm})$. Fonte: AMM, Carte e piante, M. 9.

parte, di fare ulteriormente chiarezza sulla questione della "disputa de' Risi" e, dall'altra parte, ci restituisce un'immagine del paesaggio rurale collinare e della sua organizzazione insediativa e colturale.

Il disegno, di grandi dimensioni, raffigura gli attori di questa causa ovvero il monastero di Montecassino, le universitates - i cui insediamenti, di origine medioevale, si sviluppano all'ombra delle colline, che assicurano protezione e qualità ambientale - e la risaia del Duca di Mignano, oggetto della contesa.

La rappresentazione del paesaggio esprime la forte vocazione rurale, favorita dal ricco sistema idrico e dalla vegetazione che si staglia in questo lembo di terra. La risaia diventa "la pietra angolare" da dove si sono "principiate le misure per linea retta delle distanze della terra di S. Vittore, S. Pietro Infine, Cervaro, Monte Casino e Mignano". Informazione, quest'ultima, strategica per conoscere e valutare la risaia in rapporto al territorio circostante e per rispondere in modo puntuale alla vexata quaestio della sicurezza tra il sito incriminato e i centri vicini.

La rappresentazione dettagliata degli elementi naturali e antropici, debitamente segnati nella legenda, ci restituisce l'immagine di un territorio, con una chiara marca identitaria, legata al paesaggio rurale collinare, dove le condizioni pedoclimatiche e l'importante idrografia superficiale garantivano prevalentemente semina- tivi e coltivazioni legnose agrarie. La risaia rappresenta, pertanto, una rottura della tradizione colturale locale, vista con sospetto da chi quel territorio doveva coltivarlo e proteggerlo.

I segni cartografici e i documenti archivistici, che dovevano aiutare i decisori a dirimere la questione annosa del rapporto tra le risaie e il rischio ambientale, economico e sociale, testimoniano anche il livello di conoscenze e competenze che nel principio del XVIII secolo si aveva rispetto agli effetti di questa pratica che risultò tanto complessa e ambigua da far addivenire le parti avverse ad un accordo, non decretando, di fatto, né vincitori e né vinti.

Il Duca di Mignano venne risarcito dai ricorrenti per dismettere la pratica della risicoltura che scomparve dal paesaggio di Mignano Montelungo.

Una vera e propria damnatio memoriae, come viene confermato anche dalla cartografia ufficiale dell'Istituto Geografico Militare, che non riporta alcun riferimento toponomastico a questa esperienza colturale, che è potuta riemergere (Fig. 5) grazie ai segni cartografici storici, alla documentazione archivistica e a quella memoria locale, sempre più fievole, tramandata di padre in figlio $^{14}$, di una "risiera a Mignano".

${ }^{14}$ Ringraziamo la Prof.ssa Rosalia Lisi e il Sig. Grieco Sabatino per averci guidato alla riscoperta della risaia del Duca di Mignano, attra- 


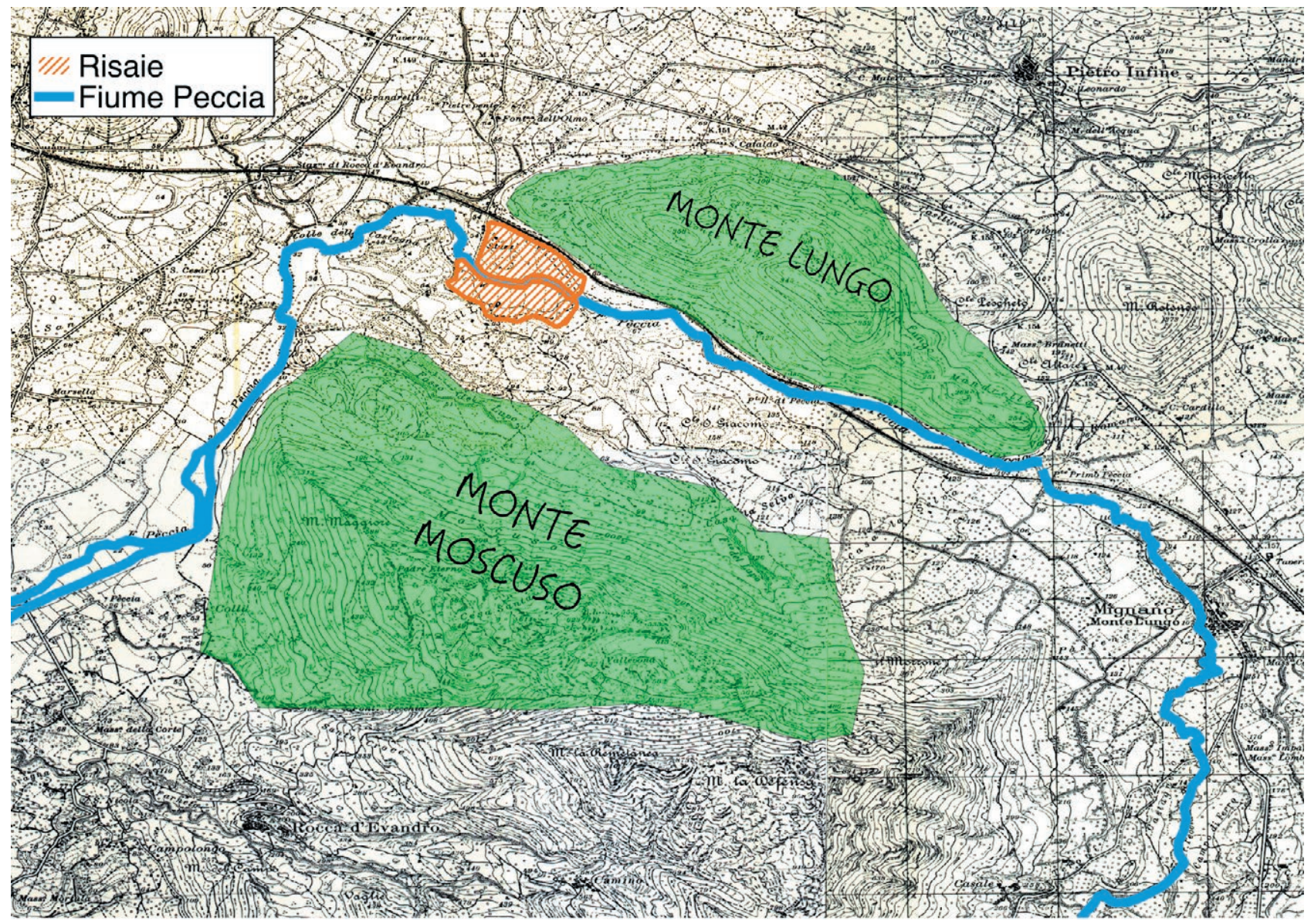

Figura 5. Gli iconemi nel paesaggio della risicoltura a Mignano Montelungo: il fiume Peccia che scorre tra Monte Lungo e Monte Moscuso e che alimentava nel XVII secolo le Risaie del Duca di Mignano, individuate grazie ai documenti d'archivio, alla cartografia storica e alla memoria locale. Fonte: elaborazione grafica a cura degli autori su base cartografica Istituto Geografico Militare (Tavolette F. 160 II N.E. [Cassino], 1942; F. 160 II S.E. [S. Ambrogio sul Garigliano], 1942; F. 161 III N.O. [Venafro], 1942; 161 III S. O. [Mignano Montelungo], 1942).

\section{Coltivazioni irrigue: il riso, la risicoltura e la sua diffusione}

Dal caso di studio è emersa, dunque, l'esistenza di coltivazioni irrigue anche nel Regno delle Due Sicilie, "laddove - scrive Bevilacqua $(2000,339)$ - le difficoltà tecniche ed economiche di utilizzazione della risorsa idrica non appaiono insormontabili, o laddove l'intervento pubblico ha creato le condizioni di economicità del suo uso [...]". A prescindere da alcuni "virtuosi" casi specifici è innegabile, tuttavia, che l'Italia meridionale sia stata caratterizzata da una minore diffusione di questo tipo di colture, in virtù di un uso delle acque meno razionale e con tecniche più obsolete (infra): il ricorso ad esse, infatti, non fu pari alle reali "potenzialità irrigue

verso la memoria storica locale che attribuisce a questo luogo il toponimo "risiera". nel territorio". Il loro uso, da parte di singoli o di comunità, fu però molto più esteso di quanto non documentato dalla letteratura dei contemporanei. Un caso particolarmente esemplificativo può essere ravvisato proprio nell'opera di diversione del fiume Peccia effettuata dal Duca di Mignano a favore della coltivazione del riso, una delle colture che, come già evidenziato, ha comportato del resto squilibri ambientali e sollecitato aspre diatribe in tutta la Penisola.

$\mathrm{Si}$ è trattato di una complessa querelle che - come già evidenziato - ha travalicato i confini locali per estendersi all'intero territorio nazionale: medici, politici, economisti, agronomi furono coinvolti, specialmente tra il Settecento e l'Ottocento, in un conflitto d'interessi senza precedenti che riguardò gli effetti della produzione risicola sulle popolazioni e sulle altre attività produttive: da una parte, i fautori dell'impianto di nuove risaie; dall'al- 
tra, gli "abolizionisti” sostenitori della salute pubblica e della salvaguardia dell'ambiente naturale.

Le risaie si diffusero certamente a ritmo sostenuto in Italia settentrionale per la presenza di numerose terre sommerse, trovando soprattutto nelle aree umide del Po il proprio contesto d'elezione ${ }^{15}$. Tuttavia, esse, nonostante la ridotta portata dei regimi fluviali, non furono così sporadiche nel Mezzogiorno. Il vantaggio economico che tale attività produttiva forniva, infatti, era considerato molto elevato: altissime erano le rese, a fronte di un ciclo biologico molto corto (Bevilacqua 2000) ${ }^{16}$. "Anche qui il riso, nonostante le continue e ripetute proibizioni dell'autorità regia, in ragione dell'intensa endemia malarica che produceva o favoriva fra le popolazioni", come nel caso esemplificativo della semina del riso a Mignano, incarnava una delle caratteristiche peculiari delle economie d'acqua (Bevilacqua 1996, 45). L'ostinazione del duca, di fatto, è la dimostrazione di quanto produttivo e redditizio fosse l'investimento nella coltivazione della pianta tanto osteggiata. Dietro la questione ambientale - particolarmente sentita negli ambienti riformisti del Regno - si celava, probabilmente, il timore dell'Abbazia di non stare al passo con le innovazioni necessarie per dinamizzare il settore agricolo, così come l'attenta lettura dei documenti ci suggerisce.

Dalla "Carta dei Prodotti Alimentari delle Provincie Continentali del Regno delle due Sicilie", (1856) di Benedetto Marzolla ${ }^{17}$, di riso ne rimane molto poco; tuttavia, risulta presente in Abruzzo Ulteriore I nel Distretto di Lanciano, in Campania in provincia di Salerno e in Calabria Citeriore nei pressi di Bisignano. Dalla sta-

\footnotetext{
${ }^{15}$ Non solo nelle paludi e nelle acque stagnanti il riso trovò le condizioni più favorevoli alla sua crescita, ma con il ricorso programmatico e razionale alle acque di scorrimento si determinò una più significativa diffusione della sua coltivazione e una maggiore condizione di salubrità. ${ }^{16}$ Nonostante l'alto costo che l'impianto di una risaia comportava, variabile ovviamente a seconda di numerosi fattori - il luogo d'impianto, la disponibilità d'acqua, la prossimità o meno di un mercato ecc. - la sua coltivazione doveva rendere molto bene. Scrive Faccini: "Tutte le fonti del tempo testimoniano comunque che il saggio del profitto ottenibile con la risicoltura era di gran lunga superiore, spesso più del doppio, a quello ottenibile con le tradizionali pratiche colturali" (Faccini 1976, 20). F. Alfonso Spagna sottolineava il fatto che la permanenza del riso, a dispetto di altre colture irrigue, dipendeva dallelevato guadagno - in Sicilia allincirca pari a 400 lire per ettaro - che non poteva essere eguagliato da nessun'altra pianta industriale irrigua (Alfonso 1877, 487).

17 "Un capolavoro di sintesi cartografica", la carta di Marzolla, come ha giustamente osservato Silvia Siniscalchi (2019), che rimanda a quell'Illuminismo meridionale dotato di uno "sguardo" operativo e concreto legato alla restituzione cartografica dei territori da governare (Manzi 1977). La carta, che utilizza i dati statistici del Ministero dell'Annona, è la raffigurazione delle produzioni e delle loro distribuzioni nel Regno delle Due Sicilie, nella parte continentale, con particolare attenzione ai prodotti agricoli, manifatturieri, dell'allevamento (Conti 2005 e 2008; Valerio, Valenti 2008; Siniscalchi 2019).
}

tistica di Raffaele Pareto, successiva di una decina di anni (1865), le risaie appaiono sempre molto contenute nei numeri, tuttavia sembrano distribuirsi in maniera più uniforme sul territorio del Regno: nelle province del centro "in riva all'Adriatico", tra cui Teramo, Chieti, Campobasso, gli ettari dedicati alla risicoltura (con "acqua perenne e con acque avventizie") sono 1105; nelle province "napoletane in riva al mare", tra cui Bari, Lecce, Potenza, Cosenza, Catanzaro, Reggio, Salerno, Napoli, Benevento, gli ettari sono in totale 12.458, cui si aggiunge Avellino con 186 ettari, per un totale complessivo di 12.644 ettari $^{18}$.

\section{Conclusioni. La vexata quaestio delle risaie tra proibizione e sostegno}

In Italia meridionale, in particolare lungo le aree costiere, dunque, "si configura la struttura della risaia ed il prodotto ottenuto entra di buon diritto nella produzione tipica di molti insediamenti" (Burri, Turchi 2010, 19). Non possiamo pensare, certamente, di essere di fronte alle ampie pianure risicole dell'Italia settentrionale, come più volte ribadito, ma si è comunque in presenza di una microeconomia d'acqua "opportunamente localizzata nel tempo e nello spazio" (Ibidem).

Le due grandi partizioni, quella del Nord e del Sud del Paese, sono accomunate dagli stessi problemi che le risaie comportano, in quanto considerate pregiudizievoli per la salute delle popolazioni, soprattutto se sviluppate nelle acque stagnanti.

Per tali ragioni si generò quel vivace e articolato dibattito - come già ampiamente descritto - che si diffuse tra il XVIII e il XIX secolo. Opinioni, idee, relazioni, perizie redatte a titolo diverso che o sostenevano la necessità della coltivazione per il vantaggio economico che ne derivava o si opponevano con tutti i mezzi affinché il coltivo venisse sospeso e la pianta estirpata del tutto. Come scrive Faccini, infatti, il dibattito sulle risaie era molto accesso a causa dell'importanza "economica e politica che la risicoltura assumeva agli occhi sia dei suoi fautori sia dei suoi avversari" (Faccini 1976, 42) ${ }^{19}$.

Questa importante diatriba ha prodotto un corposo carteggio e una significativa cartografia, i cui segni rap-

\footnotetext{
${ }^{18}$ La Sicilia, sebbene non estranea alla coltivazione del riso con un totale di 1338 ettari, in ragione del progresso dei bonificamenti e dei divieti delle autorità, cominciava a lasciare spazio ad altre colture come il cotone (Bevilacqua 2000, 66, nota 15).

${ }^{19}$ Con Faccini, si può sostenere che nelle preoccupazioni degli scrittori settecenteschi c'era forse un atteggiamento paternalistico e umanitario che si attenuò con la Restaurazione. Da questo momento la paura che serpeggia ha una chiara connotazione sociale e politica (Faccini 1976, 44).
} 
presentano una narrazione territoriale che ci restituisce la straordinarietà e il senso di molti di quei paesaggi che Ezio Burri ha voluto definire "effimeri", in altre parole: paesaggi scomparsi di cui non si ha più traccia e memoria se non nella documentazione storica e, in alcuni casi (seppure sporadici), nella tradizione alimentare (Burri 2014) ${ }^{20}$.

Con la legge del 12 giugno 1866, lo Stato unitario si pronunciò in merito alla coltivazione del riso lasciando di fatto la materia alla decisione dei singoli poteri locali, in relazione alle disposizioni di norme e di controllo. "La legge finiva col dare mano libera, nelle regioni risicole, ai grandi e medi proprietari insediati direttamente o largamente rappresentati nei Consigli provinciali"21 (Bevilacqua 2000, 57).

Fatto sta che dall'Italia meridionale la produzione del riso scomparve quasi completamente, se non del tutto. Quali le motivazioni di tale obliterazione? Probabilmente a ragion veduta Burri e Turchi $(2010,10)$ sostengono che potrebbe essersi verificata una sorta di "globalizzazione ante litteram": l'Unità nazionale e il dilatamento dei confini del governo sabaudo, con il conseguente annullamento delle dogane, hanno comportato il dilagare della concorrenza proveniente dai mercati del Nord della Penisola, certamente caratterizzato da una produzione maggiormente abbondante e da una più spiccata specializzazione a livello delle tecniche ${ }^{22}$.

\footnotetext{
20 "Parlando del riso può sembrare strano che ci si riferisca allo Stato della Chiesa ed al Regno di Napoli che notoriamente non passano per essere dei grandi produttori di riso, ma se si controllano con attenzione alcuni piatti caratteristici dei due stati pre-unitari, ci si rende perfettamente conto che in realtà il riso entra nella dieta degli abitanti, anche se certamente in misura abbastanza marginale, ma sicuramente in modo tale da divenire 'pietanza' nazionale" (Conti 2005, 92).

${ }^{21}$ È dall'età moderna che si susseguono varie ordinanze prodotte nei diversi stati italiani per limitare la coltivazione del riso. In generale, vigevano alcune misure che proibivano la risicoltura entro un determinato raggio di distanza dagli insediamenti. Tale misura poteva variare a seconda dei luoghi d'elezione: in linea di massima bisognava rispettare la distanza di due miglia dai centri abitati, misura comunque variabile in base alle disposizioni normative e ai contesti di riferimento. "La prammatica del 16 luglio 1763 - emanata nel Regno - prescriveva che la coltura del riso e le macerazioni si praticassero ad una distanza non inferiore a due miglia dai centri abitati. La legge, però, prevedeva delle eccezioni e l'articolo sesto si prestava alle più svariate interpretazioni e soprattutto ai cavilli, agli intrighi ed alle speculazioni. Tale articolo prescriveva che la coltura del riso potesse praticarsi ad una distanza inferiore alle due miglia qualora vi fosse stata «interposta» una montagna, o una vallata, o un largo fiume. Forti di quest'articolo, i coltivatori riuscivano ad evitare il provvedimento di «estirpazione», guadagnandosi la complicità degli architetti incaricati della perizia" (Gabrielli 1994, 13). Editti e regolamenti, dunque, che da nord a sud venivano evasi molto di frequente e che non facevano altro che generare contese giudiziarie, tra cui, non ultima, quella relativa al caso di studio in questione.

${ }^{22} \mathrm{~A}$ tale evidenza si può anche aggiungere la considerazione che l'ordinanza emanata da Ferdinando II (decreto del 14 gennaio 1831), con la quale si vietava la coltivazione del cereale laddove praticata in aree paludose, può aver agevolato il processo che ha portato alla definitiva
}

Di fronte a un tale fenomeno diffuso spazialmente, ci si può interrogare anche sulle ragioni sottese allo scontro/dibattito che è rimasto vivo e inalterato per circa due secoli in relazione al divieto o meno di coltivare il riso per preservare la salute delle comunità e dell'ambiente.

Come confermatoci dalla specifica letteratura (De Bernardi 1976; Faccini 1976; Bevilacqua 1986; 1989a; 1989b; 2000), la controversia affonda le sue radici negli interessi economici sollevati dalla sua coltivazione. In altri termini, si può affermare che si è trattato di uno scontro sociale e politico: da una parte l'aristocrazia legata ai tradizionali rapporti di lavoro, dall'altra la borghesia agraria fautrice dell'innovazione, la quale "si fece portavoce, attraverso i suoi intellettuali, di una sorta di apologia delle risaie" (De Bernardi 1976, 189).

Le operazioni legate al ciclo di produzione del riso richiamavano una "vasta massa di manodopera avventizia", che rappresentava "un numeroso proletariato agricolo" (Bevilacqua 2000, 42) ${ }^{23}$. Si trasformavano così i rapporti di lavoro, le relazioni sociali. "Tramontavano [...], o si sgretolavano lentamente vecchie e collaudate economie, si allentavano o si dissolvevano definitivamente consolidati rapporti colonici" (Bevilacqua 2000, 43).

La difesa ambientalista, incentrata sulla lotta alle risaie che, nel nostro caso di studio fu sostenuta dai monaci di Montecassino e, a livello generale, dagli agrari conservatori, sembrerebbe celare, dunque, una più "larga preoccupazione" di ordine sociale ed economico contro l'espandersi della coltura del riso nella sua dimensione capitalistica. I conservatori, infatti, temevano che i progressi della risicoltura, disgregando il tessuto connettivo delle relazioni sociali in agricoltura, avrebbero potuto innescare rotture con il potere (De Bernardi 1976, 190). La discussione era causata con ogni evidenza, quali che fossero le argomentazioni dietro cui si trinceravano le parti, dalle trasformazioni di carattere economico che la risicoltura comportava all'interno della struttura agraria tradizionale e all'emergere, di conseguenza, all'interno dell'aristocrazia e della borghesia di gruppi con interessi economici contrastanti (Faccini 1976, 42).

Se tale dinamica ha caratterizzato specialmente la Lombardia, il Piemonte, l'Emilia, regioni in cui la produzione del cereale avveniva in aziende di grandi

cessazione della produzione di riso nel sud della Penisola (Conti 2008). ${ }^{23}$ La mietitura del riso dava origine al più importante fenomeno sociale connesso con la risicoltura. Essa rendeva necessaria, infatti, in un ristretto periodo di tempo, la presenza di una grandissima quantità di manodopera; "manodopera che non avrebbe potuto trovare una occupazione stabile nei paesi risati durante il resto dell'anno": decine di migliaia di bracciati (Faccini 1976, 17). 
dimensioni e di più antico radicamento, è comunque in parte da estendersi anche al Regno delle Due Sicilie, seppure con le dovute differenze soprattutto per quanto attiene alle modalità di gestione dei rapporti di produzione e nella dimensione del fenomeno. Secondo Incarnato, ci si trova anche in questo caso di fronte a uno scontro tra poteri: il potere regio a favore delle classi dirigenti (l'aristocrazia locale), che volevano assicurata la rendita signorile, da una parte, e l'individualismo agrario di piccoli contadini così come di ricchi possessori (principalmente borghesi) che non vedevano di buon occhio la richiesta del sovrano della cessione del quinto del prodotto coltivato, dall'altra (Incarnato 1981) ${ }^{24}$.

Sottese, dunque, a preoccupazioni di carattere sanitario, erano le motivazioni d'ordine sociale politico ed economico che rappresentarono un problema non indifferente per un Paese che si avviava verso la grande impresa dell'Unificazione.

Laddove la risicoltura s'impianterà su scala spaziale medio-grande, cambieranno decisamente i rapporti di lavoro e di produzione, come già sostenuto, cui si aggiungerà la trasformazione anche di numerosi paesaggi che da avventizi diventeranno stabili, frutto di investimenti intensivi e impegnativi dal punto di vista tecnicoidraulico. Le risaie, man mano che si sono sviluppate e diffuse sul territorio, cambiando le loro caratteristiche originali e trasformandosi da "naturali" in "artificiali", hanno testimoniato una significativa opera di "addomesticamento" della natura (Papotti, Brusa 2008).

I paesaggi del disordine idraulico sono diventati paesaggi della razionalizzazione e sistematizzazione delle acque. Da paesaggi materiali e immateriali, al tempo stesso, a paesaggi dell'abbandono, come si è verificato in alcune regioni dell'Italia meridionale, la cui ricchezza straordinaria non si è persa del tutto, perché, inscritta nella biografia di ciascun territorio, è stata ampiamente documentata attraverso testimonianze cartografiche e testuali che ci hanno restituito un quadro delle criticità a livello territoriale e delle scelte adottate per la loro risoluzione.

In questo senso vanno lette e interpretate le relazioni, le perizie, i pareri degli esperti e la cartografia storica relativi alla controversia giudiziaria tra l'Abbazia di Montecassino in difesa dei suoi "sudditi" e il Duca di Mignano in difesa dei suoi interessi economici: una ricca documentazione, la cui analisi ci ha consentito di approfondire la conoscenza di interventi e soluzioni

\footnotetext{
${ }^{24}$ La riflessione dell'autore, sebbene focalizzata sul Teramano, provincia meridionale in cui la produzione del riso era molto praticata, può essere considerata rappresentativa per il resto del Regno, dal momento che il tema viene affrontato a una scala macroregionale, nel tentativo di sondare questioni di carattere politico-finanziario a livello complessivo.
}

adottati per risolvere problematiche di carattere sociale e ambientale in un territorio che, tuttavia, ancora agli albori dell'Ottocento mostrava i segni di una importante arretratezza nello sviluppo di tecniche irrigue più innovative.

Tale lettura ci ha offerto anche la possibilità di ricostruire i lineamenti principali di un paesaggio agrario che non solo ha lasciato tracce nei documenti testuali e nelle cartografie correlate (segni grafici, simboli, toponimi ecc.), ma anche nella memoria orale delle comunità locali. Si sono costruite così, nel tempo, solide basi affinché quei paesaggi "effimeri" non cadessero nella dimenticanza "storica", ma anzi restituissero immagini codificate tali da evocare un passato, una tradizione profondamente radicata nella coscienza individuale e collettiva.

\section{Riferimenti bibliografici}

Afan de Rivera, C. (1833). Considerazioni sui mezzi da restituire il valore proprio ai doni che ha la natura largamente conceduto al Regno delle Due Sicilie voll. I-II. Napoli, Dalla stamperia e cartiera del Fibreno.

Archivio dell'Abbazia di Montecassino (1986). In Guida Generale degli Archivi di Stato italiani. Vol. III, N-R. Roma, Ministero per i beni culturali e ambientali-Ufficio centrale per i beni archivistici, 147-149.

Bevilacqua, P. (1982). Agricoltura e storia delle campagne nel Mezzogiorno d'Italia. Studi Storici, 23 (3), 671-682.

Bevilacqua, P. (1986). Acque e bonifiche nel Mezzogiorno nella prima metà dell'Ottocento. Studi Storici, 27 (2), 335357.

Bevilacqua, P. (1989a). Acque e terre nel Regno di Napoli. In Lambiente nella storia d'Italia. Studi e immagini. Venezia, Marsilio, 73-88.

Bevilacqua, P. (a cura di). (1989b). Storia dell'agricoltura italiana in età contemporanea. Gli Spazi e paesaggi. Venezia, Marsilio, 255-318.

Bevilacqua, P. (2000). Tra Natura e Storia. Ambiente, economie, risorse in Italia. Roma, Donzelli.

Bevilacqua, P., Corona, G. (a cura di). (2000). Ambiente e risorse nel Mezzogiorno contemporaneo. Corigliano Calabro, Meridiana.

Burri, E., Turchi, M. (2010). Spunti per un'analisi dei paesaggi effimeri dell'Abruzzo: la coltivazione del riso in periodo storico tra Campli, S. Omero e Bellante. Notizie dalla Delfico, 1-3, 18-26. 
Burri, E. (2014). Il paesaggio effimero: la coltivazione del riso nell'Abruzzo pedemontano e costiero. In Bonini, G., Visentin, C. (a cura di). Paesaggi in trasformazione. Teorie e pratiche della ricerca a cinquant'anni dalla Storia del paesaggio agrario italiano di Emilio Sereni. Bologna, Editrice Compositori, 495-497.

Bustico, G. (1932). La Legislazione del riso in Italia nelle varie regioni e attraverso $i$ tempi. Novara, tip. Cattaneo.

Cazzola, F. (1987). Le bonifiche nella Valle Padana: un profilo. Rivista di storia dell'agricoltura, 27 (2), 37-66.

Ciasca, R. (1928). Storia delle bonifiche del Regno di Napoli. Bari, Laterza.

Conti, S. (2005). Alimentazione nell'Italia centromeridionale nel secolo XIX. In Palagiano, C., De Santis, G. (a cura di). Geografia dell'alimentazione. Perugia, Rux Editrice, 91-99.

Conti, S. (2008). Lagroalimentare nel Regno delle Due Sicilie in una carta di Benedetto Marzolla. In Castiello, N. (a cura di). Scritti in onore di Carmelo Formica. Napoli, Università degli Studi di Napoli Federico II.

Conti, S., Di Biasio, A. (a cura di). (2012). La terra di Lavoro nella Storia. Dalla cartografia al vedutismo. Caserta, Associazione "Roberto Almagià" - Associazione Italiana Collezionisti di Cartografia Antica.

Conti, S., Pignatelli Spinazzola, G. (2010). Le Bonifiche del Regno di Napoli nelle documentazioni cartografiche e di archivio e nella realtà odierna. In Atti della XIV Conferenza Nazionale ASITA (Brescia 9-12 novembre 2010). Milano, ASITA.

Conti, S. (2009). La toponomastica e centri abitati in Terra di Lavoro dal 1860 ad oggi. In Mascilli Migliorini, L. (a cura di). Terra di lavoro. I luoghi della storia. Avellino, Sellino, 323-334.

Corti, C. (1986). La Malaria nel Mezzogiorno Tra Otto e Novecento. In Annali della Storia d'Italia, 7. Torino, Einaudi.

D'Elia, C. (1994). Bonifiche e Stato nel Mezzogiorno (1815-1860). Napoli, ESI.

Dai Prà, E. (a cura di). (2003). APSAT9. Cartografia storica e paesaggi in Trentino: approcci geostorici. Mantova, SAP-Società archeologica.

De Bernardi, A. (1976). Risicoltura e capitalismo. Studi storici, 17 (3), 185-193.

Delfico, M., (1903). Memoria sulla coltivazione del riso nella provincia di Teramo, vol. III. Teramo, Fabbri ed. ( $1^{\mathrm{a}}$ edizione 1783).
Della Valle, C. (1956). La coltivazione del riso nell'Agro Romano. Bollettino della Società geografica italiana, serie 8, 9 (11-12), 547-52.

Di Biasio, A. (1991). Carlo Afan de Rivera e il Corpo dei Ponti e Strade. Latina, Amministrazione Provinciale.

Di Biasio, A. (1979). Gli “ordegni rustici” nell'agricoltura napoletana del primo Ottocento (una ricerca in corso). Rivista di Storia dell'Agricoltura, 19 (2), 73-142.

Fabiani, L. (1968). La Terra di S. Benedetto. Studio storico-giuridico sull'Abbazia di Montecassino dall'VIII al XIII $s e c$. , voll. I-II. Montecassino, Badia di Montecassino.

Faccini, L. (1976). Uomini e lavoro in risaia. Il dibattito sulla risicoltura nel '700 e nell'800. Milano, FrancoAngeli.

Fiengo, G. (1988). I regi Lagni e la bonifica della "Campania felix" durante il viceregno spagnolo (Italiano). Firenze, Olschki.

Foscari, G. (1992). Prassi amministrativa e attività pubblicistica a tutela del territorio: l'opera di Carlo Afan de Rivera nell'Ottocento borbonico. In Musi, A. (a cura di). Economia, società e politica del territorio nel Mezzogiorno (secc. $X V$-XIX). Salerno, Università degli Studi di Salerno.

Foscari, G. (2009). Teodoro Monticelli: e l' "economia delle acque" nel Mezzogiorno moderno. Storiografia, scienze ambientali, ecologismo. Salerno, Edisud.

Franzese, P. (2020). Caserta e la sua provincia (18182018). Il problema delle fonti archivistiche. In Brevetti, G., Sodano, G., De Lorenzo, R., Franzese, P. (a cura di), 1818-2018. Caserta e la sua provincia, Santa Maria Capua Vetere (CE), Dipartimento di Lettere e Beni culturali, Università degli Studi della Campania "Luigi Vanvitelli" (“Quaderni di Polygraphia”, 1), 17-26.

Gabrieli, G. (1994). Le risaie di Rocca D'Evandro. Rassegna storica dei comuni, 20 (72-73), 13-18.

Gabrieli, G. (1995). Le risaie di Rocca D'Evandro. Rassegna storica dei comuni, 21 (76-77), 38-45.

Gambi, L. (1989). Una patria artificiale nata governando le acque. In L'ambiente nella storia d'Italia. Studi e immagini. Venezia, Marsilio, 57-72.

Giustiniani, L. (1803). Dizionario geografico-ragionato del Regno di Napoli di Lorenzo Giustiniani a Sua Maestà Ferdinando IV Re delle due Sicilie, tomo VI. Napoli, Stamperia di Giovanni de Bonis.

Incarnato, G. (1981), Grano, Riso... e Riforme nel teramano nella seconda metà del secolo XVIII. In Massafra, A. (a cura di). Problemi di storia nelle campagne meridionali nell'età moderna e contemporanea. Bari, Dedalo, 353-371. 
Manzi, E. (1987). Illuminismo lombardo, illuminismo napoletano. Cartografia e territorio. Rivista Geografica Italiana, 94, 337-359.

Marzolla, B. (1856). Carta dei Prodotti Alimentari delle Provincie Continentali del Regno delle due Sicilie, Napoli, Real Ministero di Stato per l'Interno.

Messedaglia, L. (1938). Per la storia delle nostre piante alimentari: il riso. Siena, tip. S. Bernardino.

Ministero di Agricoltura, Industria e Commercio, Direzione generale dell'agricoltura (1889). Monografia statistica ed agraria sulla coltivazione del riso in Italia, Roma, tip. Eredi Botta.

Novelli, N., Sampietro, G. (1925). La risicoltura in Italia. In Direzione generale della Sanità pubblica, La risicoltura e la malaria nelle zone risicole d'Italia. Roma, Provveditorato generale dello Stato.

Papotti, D., Brusa, C. (2008). Risaie in Italia. In Angelini, R., Ferrero, A., Ponti, I. (a cura di). Il riso. Milano, Bayer CropScience, 200-235.

Pareto, R. (1865). Sulle bonificazioni, risaie, irrigazioni del Regno d'Italia. Milano, Tipografia e Litografia degli Ingegneri.

Siniscalchi, S. (2019). La carta "alimentare" di Benedetto Marzolla (1856) fra prodotti tipici e risorse territoriali. Bollettino della Associazione Italiana di Cartografia, 167, 68-78.

Spagna, F. A. (1877). Trattato d'idraulica. Palermo, Tip. P. Montaina \& Comp. già del Giornale di Sicilia.

Valerio, V., Valenti, P. (2008). Benedetto Marzolla. Brindisino, Geografo e Cartografo dell'Ottocento europeo. Manduria, Barbieri Selvaggi.

\section{Fonti manoscritte}

Archivio Abbazia di Montecassino (AAM), Capsula CXXXIV, fascc. II, III.

Archivio Abbazia di Montecassino, Piante e Mappe. 